\title{
First-Principles Studies Of Ionic And Electronic Transport In Palladium Hydride
}

\author{
N. LUO AND G. H. MILEY \\ Department of Nuclear, Plasma and Radiological Engineering, University of Illinois. \\ 103 S. Goodwin, \\ Urbana, IL 61801, USA \\ E-mail:nluo@uiuc.edu
}

\begin{abstract}
The transport properties of palladium hydride/deuteride are investigated using state-of-the-art density functional computational tools. This aspect of loaded hydride is important in that the resistance-loading curve is often used as a diagnostic in experiments to estimate the loading ratio. Understanding transport provides other important insights into some features of the nuclear reaction mechanism. This research involves both ionic and electronic degrees of freedom. For the ionic part, the focus is on the charge state of hydrogen/deuteron, in both a static and a dynamic (hopping) situation. Experiments show that hydrogen hops as fractional-charged positive ion in Pd lattice $[1,2]$ while previous band structure calculations [3-5] always gave a negatively charged $\mathrm{H}$ in the ground state. This discrepancy is addressed in the current research and some results are already published [6]. The collective motion of ions is studied in the phonon structure and electron-phonon coupling constant using a perturbation density functional theory. The electronic part focuses on the density of state and the Fermi surface, which when combined with the electron-phonon coupling constant, determine the temperature and the H/D loading dependant resistivity curves. Our numerical results [7] qualitatively match experimental trends. The possible connections between this transport theory/mechanism and the non-equilibrium conditions required for excess heat are discussed.
\end{abstract}

\section{Introduction}

The palladium hydride $\left(\mathrm{PdH}_{x}\right)$ system has generated much interest among a variety of scientific disciplines, such as fuel cell research, superconductivity, and low energy nuclear reactions. It poses unique problems un-common to ordinary metals, insulators or semiconductors. For example a hydrogen atom is a stronger attractor of electron than most metal atoms. Therefore the bonding nature between hydrogen and metals is different from a metal bond.

The transport properties of $\mathrm{PdH}$ are one of the most perplexing. Apart from the surprising superconductor transition when hydrogen is loaded into Pd and the associated inverse isotope effect, the normal state electric resistivity $\rho$ itself has not been well understood so far. As seen from Fig. 1, the resistivity first increases with the $\mathrm{H}$ fraction $x$ at the low loading regime. It then reaches a maximum at $x \sim 0.6$, with a surprising drop following at the further increase of $x$. More surprising is the report of an instability oscillation at very high hydrogen loading. An oscillation in $\rho$ was reported earlier [7] although more experiments are needed to make certain its existence. The oscillation indicates some instability in the charge transport. 


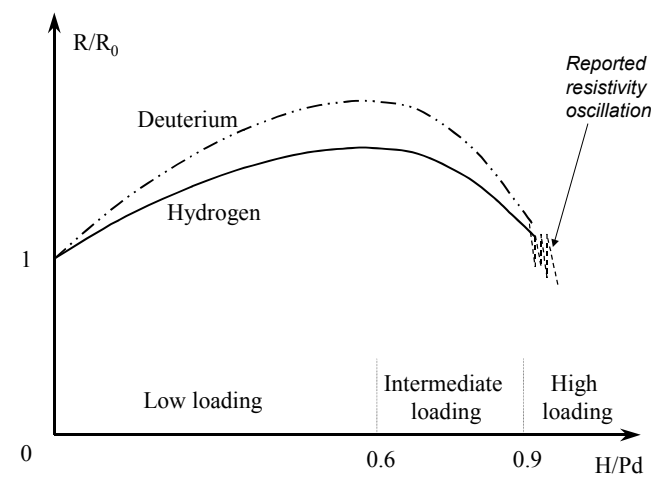

Figure 1. Normalized resistance $R / R_{0}$ vs loading, indicating the change in resistivity. $R_{0}$ is the resistance of pure Pd. The solid curve is for hydrogen loading with the dot-dashed one for deuterium.

The instability is of particular interest since it could result in coherent oscillations that have deep implications for LENR research. It is now well accepted that the reported excess heat is not likely from a deuteron/palladium system at equilibrium, but such an instability could push the system out of equilibrium. Although the reported oscillation is only preliminary, the authors believe it must be associated with the resistivity drop at high $x$. The major focus of this paper is therefore to understand the mechanism that gives rise to the characteristic $\rho-x$ curve.

\section{Electronic Structure of PdH and ionic transport of $\mathbf{H}$}

First a few remarks on the electronic structure of $\mathrm{PdH}$ are worth making. For the pure $\mathrm{Pd}$ metal, there are 3 bands of chiefly $d$-character crossing the Fermi level. This raises the possibility of inter-band electron-electron (e-e) scattering, which would significantly contribute to the resistivity. For the purpose of simplicity, e-e scattering is not considered in this paper. Instead, only electron-phonon scattering will be included.

The transport of ionic H in Pd has been quite puzzling. Drift experiments [1-2] suggest that $\mathrm{H}$ drifts in $\mathrm{Pd}$ in the form of a positive ion with a fractional charge number ranging from +0.4 to +0.7 . These observations strongly support the notion of a proton model in which the hydrogen is viewed as donating its electron to the unfilled $\mathrm{Pd} d$-shell. However, such a proton model seems oversimplified from other experiments like the electronic specific heat. Moreover from a chemistry point of view, $\mathrm{H}$ can in principle acquire an electron from the host Pd because its electron affinity and ionization energy are larger than those of Pd. Therefore, band structure calculations [3-5] have been utilized to elucidate the charge state of $\mathrm{H}$ in $\mathrm{Pd}$. The general conclusion is that $\mathrm{H}$ is slightly negatively charged in spite of the filling up of the $\mathrm{Pd} d$-bands. Without further study, this result has appeared to contradict observations made in the $\mathrm{H}$ drifting experiment.

To understand the effective positive charge of drift experiments and the negatively charged $\mathrm{H}$ ion found in previous band-structure calculations, the authors improved the calculation with the latest computational tools. The studies explicitly include hydrogen locations not previously studied, such as the bridging site indicated in Fig. 2 


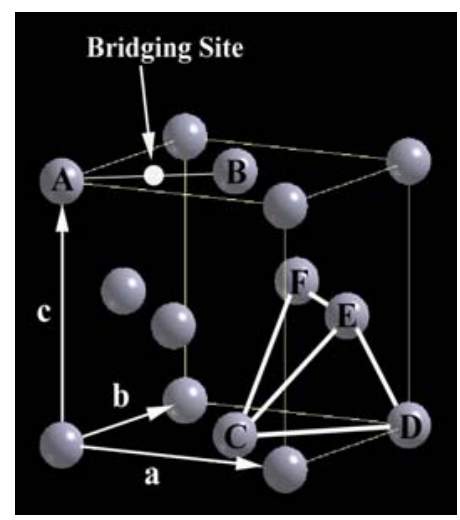

Figure 2. The definition of the bridging site in Pd lattice. It is the mid-point between two nearest neighbored Pd atoms, such as A and B.

Using a full-potential linearized augmented plane wave (FLAPW) package, WIEN2K [8], the authors were able to explore the charge distribution near $\mathrm{H}$ at different crystalline sites. The calculation in general confirms the notion of a negatively charged $\mathrm{H}$ ion in $\mathrm{Pd}$, as obtained from previous studies. Moreover, by considering sites important to the dynamic hopping process, such as the bridging site, the positively charged $\mathrm{H}$ ion seen in drift experiments can now be understood.

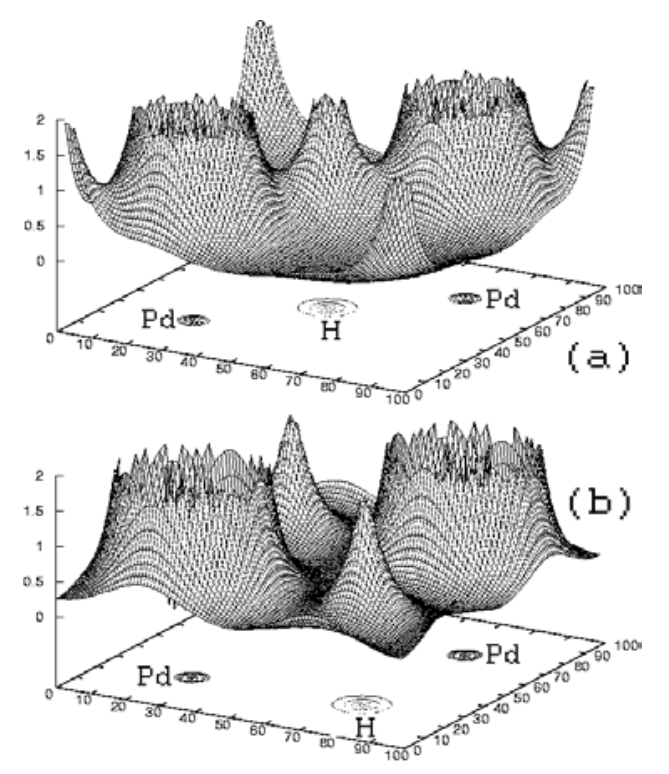

Figure 3. The charge distribution around (a) bridging-site hydrogen, and (b) octahedral hydrogen in (100) plane of a hypothetical PdH lattice. The horizontal axes are labeled in percentage of the FCC cell lengths in (100) planes.

The charge distributions around a bridging $\mathrm{H}$ and the ordinary octahedral $\mathrm{H}$ are compared in Fig. 3. More electron charge is clearly seen around the $\mathrm{H}$ in the former location. In fact the total charge in the $\mathrm{H}$ muffin-tin sphere of $r=1.5 \mathrm{bohr}$ is respectively 1.81 and 1.13 for the two cases.

Consequently the fractional positive charge can be interpreted as a dynamic property. When an electric field is applied to an octahedral $\mathrm{H}$, the proton hopping process will be assisted in the direction of the field. Therefore, the hydrogen drift is characterized as that of a proton, i.e., a +1 charge, at the octahedral site. However, in hopping from one octahedral site to the next, the proton likely follows a path through the bridging site, and the charge state 
there affects the average observed charge of the hydrogen in a drift experiment. An effective electron cloud of -0.6 is obtained from the total charge difference at the octahedral and the bridging sites as the $\mathrm{H}$ goes through the bridging region. Hence the net time averaged proton charge manifested in this drift path is 1 minus $0.6 \sim 0.4$, in qualitative agreement with drift experiment results.

\section{Resistivity from electron-phonon interaction}

Previous studies had investigated the phonon dispersion and density of state of $\mathrm{Pd}$ and $\mathrm{PdH}$. Here we extend such studies to include a key part of the problem: the electron-phonon coupling constant, at different loading $x$. Our objective is to explain the experimental $\rho-x$ curve, through a first-principles study of the electron-phonon coupling strength.

\subsection{Theoretical Basis of Electric Resistivity}

Without considering any interband e-e scattering, the resistivity of a metal is given by the Bloch-Gruneisen formula,

$$
\rho(T)=\rho_{0}+\lambda_{t r} \frac{4 \pi}{\omega_{p, D}^{2}} \frac{128 \pi\left(k_{B} T\right)^{5}}{\left(k_{B} \Theta_{D}\right)^{4}} \int_{0}^{\Theta_{D} / 2 T} \frac{x^{5}}{\sinh ^{2} x^{x}}(d)
$$

where $\lambda_{t r}$ is the dimensionless electron-phonon coupling constant. $\rho_{0}$ is the residual resistivity at absolute temperature $0 \mathrm{~K}$, attributed to scattering from lattice impurities. $\Theta_{D} \propto \sqrt{\text { bulk modulus/mass density }}$ is Debye temperature, readily calculated if bulk modulus $K$ is known. $\omega_{p, D}^{2}=\frac{4 \pi n e^{2}}{m}$, the plasma frequency, is actually the measurement of the number of band carriers $n$, including both electron and hole in the case of Pd. Note that in Eq. (1), $n$ and $m$, the density and the band mass of relevant carriers, can be calculated from the band structures at and near the Fermi surface. Such calculations are available from a typical electronic- structure package. The bulk modulus $K$ is, strictly speaking, a function of temperature, but here it can be simply approximated by $\frac{\partial U}{\partial V}$, where $U$ is the total energy and $V$ the crystal volume. Therefore, a straightforward volume-dependent total energy study using WIEN2K and other tools like CASTEP determines $K$.

\subsection{Calculation of Electron-Phonon Coupling}

The eigenvalues of bands that cross the Fermi surface were calculated for $n$ and $m$ at special $k$ points in the irreducible Brillouin zone (IBZ). The band mass tensor was then calculated by an interpolation scheme near the Fermi surface. Next, the IBZ $k$ points were generalized to the whole $\mathrm{BZ}$ in order to find out the effective carrier number of the relevant bands. The resulting $\hbar \omega_{p, D}$ and $\Theta_{D}$ are listed in the first two data rows of Table 1. 
Table 1. Plasma frequency, Debye temperature and electron-phonon coupling constant at different $\mathrm{H}$ loadings.

\begin{tabular}{|l|l|l|l|l|}
\hline & $\mathrm{Pd}$ & $\mathrm{PdH}_{0.25}$ & $\mathrm{PdH}_{0.5}$ & $\mathrm{PdH}$ \\
\hline$\hbar \omega_{p, D}(\mathrm{eV})$ & 7.68 & 7.34 & 7.03 & 7.80 \\
$\Theta_{D}(\mathrm{~K})$ & 281 & 293 & 304 & 328 \\
$\lambda_{t r}$ & 0.47 & 0.51 & 0.52 & 0.54 \\
\hline
\end{tabular}

The electron-phonon coupling is treated perturbatively using the Planewave Self Consistent Field (PWSCF) package [9]. The norm-conserved pseudopotential (PP) of Pd used in the calculation is obtained from Paolo Giannozzi's PP code [10]. To ensure the transferability, the PP was tuned by calculating the band structure of Pd using PWSCF until a satisfactory comparison with the WIEN2K band is achieved. The result is given in the last row of Table 1.

The trend seen in electron-phonon coupling versus loading can be understood from other experimental facts. Pure $\mathrm{Pd}$ is not superconductive but stoichiometric $\mathrm{PdH}$ has a critical temperature $T_{c}$ of $9 \mathrm{~K}$. This physical change indicates stronger electron-phonon interaction at higher $\mathrm{H}$ loading. Therefore the theoretical $\lambda_{t r}$ result given in Table 1 generally follows the trend for the superconducting transition temperature.

The plasma frequency $\Theta_{D}$, i.e., the total number of charge carriers, keeps decreasing up to $x \sim 0.5$, then a rise occurs as $x$ approaches 1 . This can be understood from the band filling process when $\mathrm{H}$ is added to $\mathrm{Pd}$. Palladium has three predominantly $d$-bands traversing the Fermi level. The 4-th and 5-th bands have hole-like Fermi surfaces while that of the 6-th is electron-like. At low loading, the added electrons from hydrogen mainly fill up the two hole bands, resulting in a decrease in the number of holes, which outpaces the increase in the electron number of the 6 -th band. Therefore a reduction in the total number of current carriers happens, causing a decreasing plasma frequency with increasing loading. At $x=0.6$, however, the two lower bands are now largely full. Then the increase in the electron number of the 6 -th band more than offsets the loss in the hole number. Therefore $\Theta_{D}$ begins to increase.

Substituting the calculated parameters into Eq. (1), the temperature dependent resistivity is then derived for various loadings as shown in Fig. 4. The linear temperature dependence is the characteristic of a phonon-induced resistivity when temperature is on the order of a few hundred degrees Kelvin.

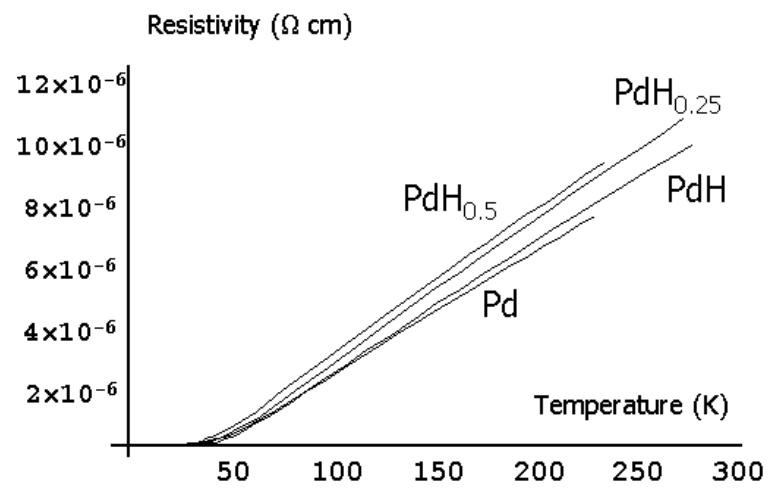


Alternatively, the normalized loading-dependent resistance at the room temperature is given in Fig. 5. The absolute values of theoretical results are well below the experimental ones. It is not a surprise considering the additional scattering from the $\mathrm{H}$-induced disorder and the inter-band electron-electron scattering not included in the calculation.

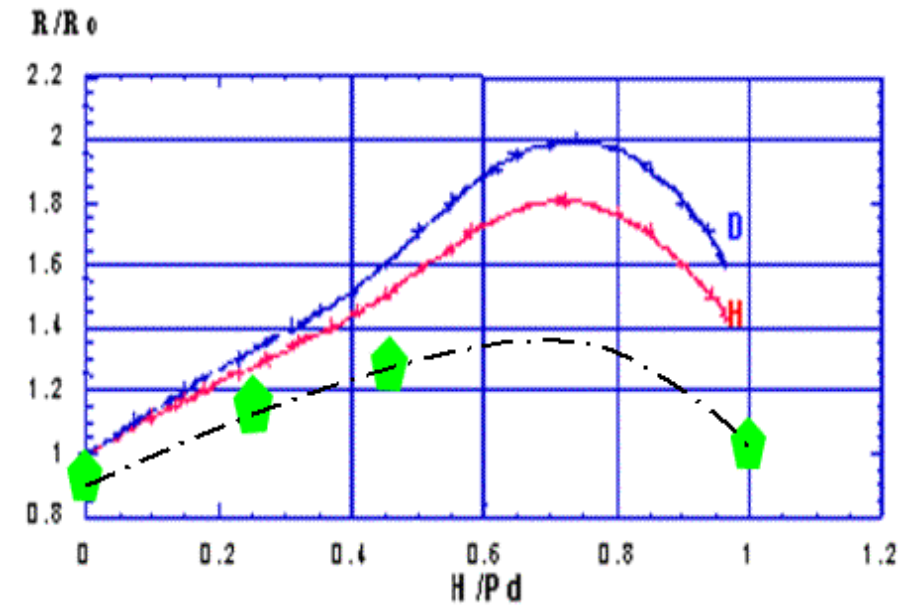

Figure 3. The normalized resistivity at different $\mathrm{H}$ loadings. The resistivity (resistance) is normalized to that of pure Pd at $300 \mathrm{~K}$. The red and blue curves are from experiment. Theoretical results are given in pentagons. The black dot-dashed line is the theoretical least-square fit.

\section{Conclusions}

The $\mathrm{H}$ loading dependent resistivity of $\mathrm{PdH}$ is calculated for the first time using firstprinciples methods. The characteristic "peak" in the $\rho$-x curve is now explained in terms of fundamental quantities: electron-phonon coupling and the filling-up of Pd hole-like bands. The positive charge for $\mathrm{H}$ ions observed in drift experiments is explained as a dynamic property associated with different electron accumulation near $\mathrm{H}$ at various sites during the hopping process.

\section{Acknowledgments}

This research was supported by Lattice Energy, LLC. Helpful discussions with A. Lipson are greatly appreciated.

\section{References}

1. H. Wipf, in: Hydrogen in metals II (Eds. G. Alefeld, J. Volk1 ), Springer-Verlag, Berlin, 1978, p. 273.

2. R. Pietrzak, R. Szatanik and M. Szuszkiewicz, J. Alloys Comp. 282 (1999) 130.

3. A.C. Switendick, Ber. Bunsenges. Phys. Chem. 76 (1972) 535.

4. D.A. Papaconstantopoulos, B.M. Klein, J.S. Faulkner and L.L. Boyer, Phys. Rev. B 18 (1978) 2784.

5. M. Gupta, A.J. Freeman, Phys. Rev. B 17 (1978) 3029.

6. N. Luo, G. H. Miley and A. G. Lipson, Applied Surface Science, 219 (2003) 167.

7. G.H. Miley, E. Selvaggi et al., ICCF-8 Proceedings, Lerici, Italy (2000) p. 419.

8. WIEN2K, http://www.wien2k.at 
9. PWSCF, http://www.pwscf.org

10. http://www.nest.sns.it/ giannozz/software.html 